\title{
Dynamics of migrating sand dunes interacting with obstacles
}

\author{
Karol A. Bacik, ${ }^{1, *}$ Priscilla Canizares, ${ }^{2}$ Colm-cille P. Caulfield, ${ }^{3,1}$ \\ Michael J. Williams, ${ }^{2}$ and Nathalie M. Vriend ${ }^{3,1,4}$ \\ ${ }^{1}$ Department of Applied Mathematics 8 Theoretical Physics, \\ University of Cambridge, Centre for Mathematical Sciences, \\ Wilberforce Road, Cambridge CB3 0WA, United Kingdom \\ ${ }^{2}$ Schlumberger Cambridge Research, High Cross, \\ Madingley Rd, Cambridge CB3 OEL, United Kingdom \\ ${ }^{3}$ BP Institute, University of Cambridge, Madingley Rise, \\ Madingley Road, Cambridge CB3 OEZ, United Kingdom \\ ${ }^{4}$ Department of Earth Sciences, University of Cambridge, \\ Downing Street, Cambridge CB2 3EQ, United Kingdom
}

(Dated: October 5, 2021)

\begin{abstract}
Wind- and water-driven migrating sand dunes frequently interact with elevated natural and manmade topographical features. The dune-obstacle interaction can alter the migrating behaviour of the dune and, depending on the nature of the obstacle, it may generate various societal and technological risks. Here we study the problem of dune-obstacle interaction in a paradigmatic quasi-2D domain realised in a subaqueous laboratory experiment. Generically, dunes interact with obstacles either by 'crossing' over the obstacle or by being 'trapped'. We describe how the selection of these two distinct dynamical behaviours depends on the size and shape of the obstacle, focusing in particular on the fluid flow in the immediate vicinity of the obstacle. Specifically, we perform a modal decomposition of the measured flow field and we discover that the outcome of the duneobstacle interaction is closely related to the flow structure above the obstacle.
\end{abstract}

\footnotetext{
${ }^{1}$ Present address: Centre for Networks and Collective Behaviour, Department of Mathematical Sciences, University of Bath, Bath, BA2 7AY, United Kingdom

*kab81@cam.ac.uk
} 


\section{INTRODUCTION}

When a pile of loose sediment is exposed to sufficiently energetic wind or water current, it spontaneously transforms into a migrating sand dune [1-3]. The course of a migrating dune can be interrupted by a number of mobile and immobile topographical factors. First of all, it has been shown that dunes can interact and collide with other sand dunes [4-8]. Secondly, dunes can interact with various fixed natural and human-made topographical obstacles. Indeed, dune invasion is one of the main hazards associated with infrastructure erected in sedimentary environments $[2,9]$. Aeolian dunes notoriously bury roads, rail-tracks, or even buildings [10-12]. Under water, dune migration entails dredging of navigation channels and compromises the safety of subsea cables and pipelines [13]. Despite its direct societal relevance, the problem of dune-obstacle interaction has apparently received relatively little attention in the physical literature. To bridge this gap, in this article we study experimentally an idealised quasi-2D interaction scenario whereby a migrating subaqueous dune interacts with a solid obstacle.

We consider a variety of obstacles, with differing cross-sectional shapes and areas. Generically, two qualitatively different dynamical behaviours are possible. For 'small' obstacles, sufficient amount of the granular material is able to 'cross' over the obstacle, so that a dune reconstitutes on the downstream side of the obstacle, and migrates away. Depending on the cross-sectional area and geometric shape of the obstacle, varying quantity of the granular material is trapped in the immediate vicinity of the obstacle. Indeed, for sufficiently 'large' obstacles, no dune reforms on the downstream side, and so the dune in its entirety may be thought of as being 'trapped' by the obstacle. A key aim of our study is to identify and understand the controlling properties which determine whether the dune (at least partially) crosses or is (entirely) trapped after interaction with a particular obstacle. We focus on quasi-2D subaqueous sand dunes realised in a an annular experimental set-up. The closest natural analogue of a quasi-2D sand dune is a transverse dune, but it can also be thought of as a cross-section through a more complicated topography.

We begin our discussion by introducing our experimental apparatus (Sec. II). Subsequently, we use an illustrative example to discuss general features of the dune-obstacle in-

teraction (Sec. III A) and flow around the obstacle (Sec. III B). In Section III C we describe qualitatively how the obstacle's shape and size, as well as the background flow velocity, 
impact the outcome of the dune-obstacle interaction, drawing distinction between 'crossing' and 'trapped' dunes. In Section IIID we find a quantitative link between the modal structure of the flow in the immediate vicinity of the obstacle and the dividing line in parameter space between crossing and trapped dune dynamics. Finally, we draw our conclusions in Section IV.

\section{METHODS}

\section{A. Experimental set-up}

In our studies we deploy an annular flume of outer radius $R=0.97 \mathrm{~m}$, filled with water up to $H_{w}=0.45 \mathrm{~m}$ (Fig. 1(a)) [4]. The working section is a $W=0.09 \mathrm{~m}$ wide channel which mimics a straight channel with streamwise periodic boundary condition $(W / R=9 / 97 \ll 1)$. The flow in the channel is induced by an assembly of twelve paddles of height $H_{p}=0.1 \mathrm{~m}$ which rotate counter-clockwise at angular speed $\Omega_{p}$. The paddles are partially submerged with the upper tip protruding $0.01 \mathrm{~m}$ above the free surface. The flume rests on a turntable, which rotates clockwise at speed $\Omega_{t}$. The primary objective of this counter-rotation is to create spanwise invariant quasi-2D bedforms by balancing the effects of the centrifugal force and secondary flows [14]. The flow is fully turbulent with channel-based Reynolds number $\operatorname{Re} \sim 10^{5}$.

The dunes we study are made of spherical glass beads (Sigmund Lindner, Deco Beads 4504-187-LS) of nominal density $\rho_{p}=2500 \mathrm{kgm}^{-3}$ and random packing fraction $\eta=0.6$. The mean particle diameter $\bar{d}=1.17 \mathrm{~mm}$ and the material is close-to-monodisperse with virtually no particles with $d<1.0 \mathrm{~mm}$ or $d>1.4 \mathrm{~mm}$. Each of the dunes initially contains $M=2 \mathrm{~kg}$, which corresponds to a cross-section area

$$
A=\frac{M}{\rho_{p} \eta W}=0.0147 \mathrm{~m}^{2} .
$$

Initially, sediment is arranged into a quasi-triangular heap with both sides held at the angle of repose $\beta \approx 20^{\circ}$. The flow spin-up time is of order 60 seconds and the pile moulds into its characteristic asymmetrical dune shape soon after.

Our obstacles are polystyrene cylinders and prisms wedged in at the bottom of the channel. In total we use five different shapes of different sizes - all of them are defined in Fig. 1(b). To prevent deterioration, we cover them with adhesive tape, so their surface is 


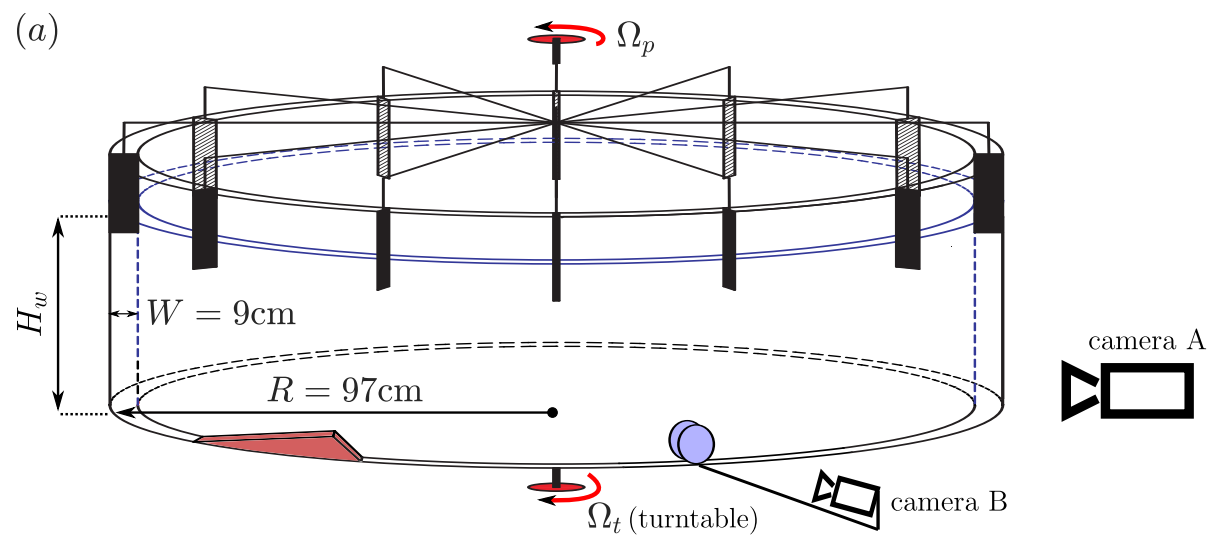

$(b)$
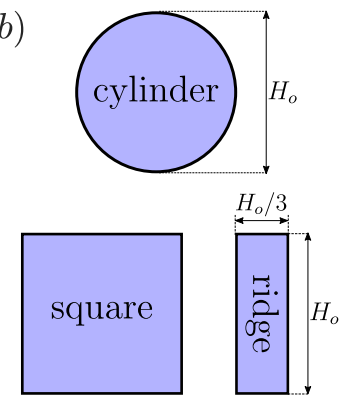

$(c)$
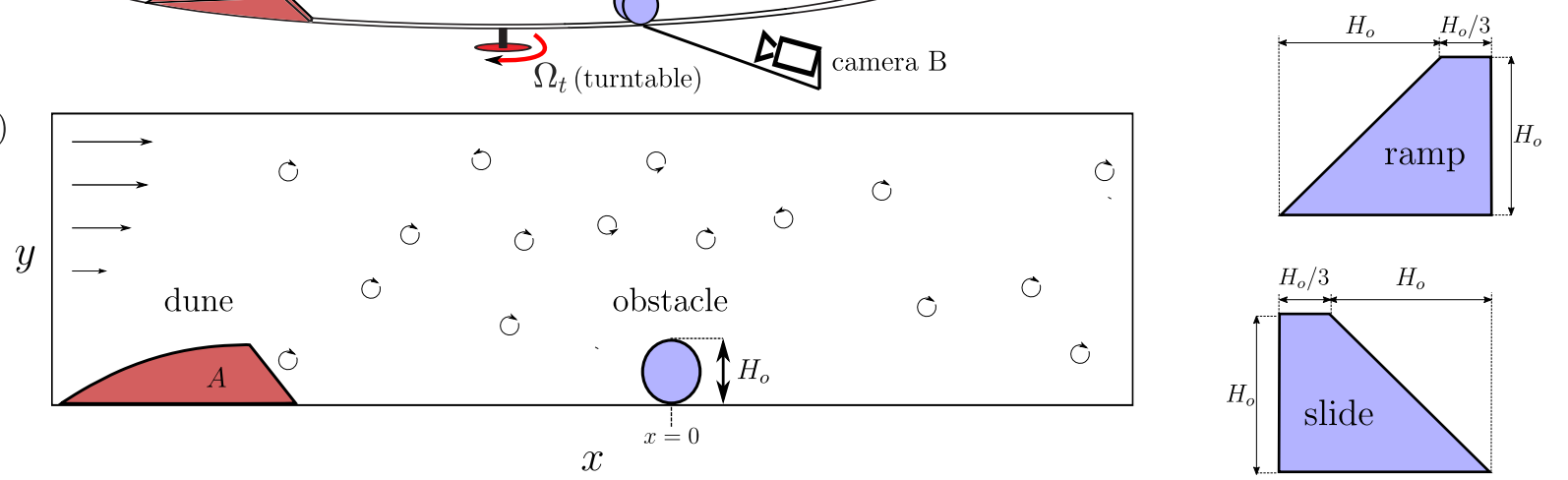

Figure 1. (a) Experimental set-up. Annular flume with a $W=9 \mathrm{~cm}$ wide working section filled with water and sediment. The motion of water is induced by an assembly of paddles rotating counter-clockwise at angular speed $\Omega_{p}$ and the dominant direction of the flow is from the dune (red) to the obstacle (blue). The flume rests on a turntable, which rotates clockwise at speed $\Omega_{t}$. The data is primarily collected with a stationary camera (A), but the sediment dynamics near the obstacle can be also visualised with an additional co-rotating camera (B) mounted to the turntable. (b) Obstacles Ridge - the cross-section is a rectangle with aspect ratio 1:3. Ramp - ridge with an upstream isosceles right triangle extension. Slide - mirror image of the ramp. In practice, both ramp and the slide are composed of two adjoined detachable blocks. (c) System set-up. In interpreting out results, we abstract the system as quasi-2D with a migrating dune of cross-section area $A$ encroaching on an obstacle of height $H_{o}$. The origin of the coordinate system is chosen so that the centroid of the obstacle is at $x=0$.

always smooth. In total, we conducted 15 different experiments with different obstacles and different flow rates, all of which are summarised in Table I. For each of these settings we also performed complementary flow imaging experiments (Sec. II C). 
obstacle shape $\left|H_{p}(\mathrm{~cm})\right| \Omega_{\text {tot }}(\mathrm{rpm})\left|\Omega_{t}(\mathrm{rpm})\right| \Omega_{p}(\mathrm{rpm}) \mid$ duration $(\mathrm{min})\left|1 / f_{\text {rec }}(\mathrm{rev})\right|$ outcome

\begin{tabular}{|c|c|c|c|c|c|c|c|c|}
\hline 1. & cylinder & 3 & 13.5 & -4.8 & 8.7 & 90 & 1 & cross \\
\hline 2. & cylinder & 6 & 13.5 & -4.8 & 8.7 & 90 & 1 & cross \\
\hline 3. & cylinder & 9 & 13.5 & -4.8 & 8.7 & 90 & 1 & trap \\
\hline 4. & square & 3 & 13.5 & -4.8 & 8.7 & 90 & 1 & cross \\
\hline 5. & square & 6 & 10.5 & -3.8 & 6.7 & 355 & 5 & cross \\
\hline 6. & square & 6 & 11.5 & -4.1 & 7.4 & 190 & 5 & cross \\
\hline 7. & square & 6 & 13.5 & -4.8 & 8.7 & 90 & 1 & cross \\
\hline 8. & square & 6 & 15.5 & -5.4 & 10.1 & 60 & 1 & cross \\
\hline 9. & square & 9 & 13.5 & -4.8 & 8.7 & 90 & 1 & trap \\
\hline 10. & ridge & 6 & 11.5 & -4.1 & 7.4 & 150 & 5 & trap \\
\hline 11. & ridge & 6 & 13.5 & -4.8 & 8.7 & 90 & 1 & trap \\
\hline 12. & ridge & 6 & 15.5 & -5.4 & 10.1 & 30 & 1 & trap \\
\hline 13. & ramp & 6 & 13.5 & -4.8 & 8.7 & 90 & 1 & cross \\
\hline 14. & slide & 6 & 10.5 & -3.8 & 6.7 & 345 & 5 & cross \\
\hline 15. & slide & 6 & 13.5 & -4.8 & 8.7 & 90 & 1 & cross \\
\hline
\end{tabular}

Table I. Experiments. A summary of all the experiments discussed in this paper. Note that the counter-rotation ratio $\left|\Omega_{p} / \Omega_{t}\right|$ is tuned empirically for each experiment to ensure a quasi-2D profile of the sedimentary bed [14]. The penultimate column, $f_{r e c}=1 / n$ signifies that we made a full scan of the topography every $n$ revolutions of the turntable.

\section{B. Topographical measurements}

An ISVI IC-X12CXP camera with a $32 \mathrm{~mm}$ Nikon lens is positioned approximately $1.5 \mathrm{~m}$ away from the flume and aimed at its central axis (camera A in Fig. 1(a)). Its field of view is a tall rectangle with a resolution of $1280 \mathrm{px} \times 20 \mathrm{px}$, corresponding to a region of approximately $170 \mathrm{~mm} \times 3 \mathrm{~mm}$ at the outer radius of the flume, which is the location where the image is taken. The frame rate is set to $200 \mathrm{~Hz}$. Appropriate uniform back-lighting is provided by a cylindrical LED panel inside the annulus.

As the system rotates, every point along the circumference appears periodically in the field of view. Critically, the rotation speed is significantly faster than the speed of dune 
migration, so we can track the evolution of the system by reconstructing the topography of the sedimentary bed at every revolution of the turntable. Continuous recordings are not feasible when the experiment is longer than 90 minutes, so in some of our experiments we record only 1 in 5 revolutions (penultimate column of Tab. I). By using a light-intensity threshold, we identify the position of the sediment-water interface along the central pixel in each frame and the data are later post-processed with a median filter. Due to flume imperfections, topography measurements near the bottom are noisy, so we disregard all features with topography height $h<1.6 \mathrm{~cm}$.

\section{Flow imaging}

Even though the curvature and rotation of our experiment hinder precise flow imaging, we can approximate the mean velocity field using Particle Tracking Velocimetry (PTV). To this end, we use a high-speed camera Phantom v2012 with an 85mm Nikon lens positioned in the same location as the ISVI camera described in Sec. II B (camera A in Fig. 1(a)), recording with a frame rate $2000 \mathrm{fps}$. Its field of view is also a tall rectangle $(800 \mathrm{px} \times 128 \mathrm{px})$, which corresponds to a region of approximate size $300 \mathrm{~mm} \times 6 \mathrm{~mm}$.

All of our flow imaging experiments were conducted without sediment, but the flow was populated with neutrally buoyant Pliolite VTAC particles. For each setting (Tab. I), we recorded 25 videos capturing the flow in a certain fixed region in the vicinity of the obstacle. Several of them (no more than 5 per setting) could not be used due to file corruption. From each video we construct an instantaneous width-averaged Eulerian velocity using the Digiflow software package [15]. Finally, by averaging the images we construct an ensembleand depth-averaged velocity field $(\bar{U}(x, y), \bar{V}(x, y))$ (c.f. Sec. III B). We use the azimuthal component of this velocity field $\bar{U}(x, y)$ to quantify various features of the flow below, in Sec. III B.

\section{Qualitative visualisation of sediment transport}

Our set-up also gives the opportunity of filming the bed with a co-rotating camera mounted $7 \mathrm{~cm}$ away from the outer wall of the flume looking inwards at the channel (Camera B in Fig. 1(a)). The device we use is a compact battery-operated travel camera (Lumix 


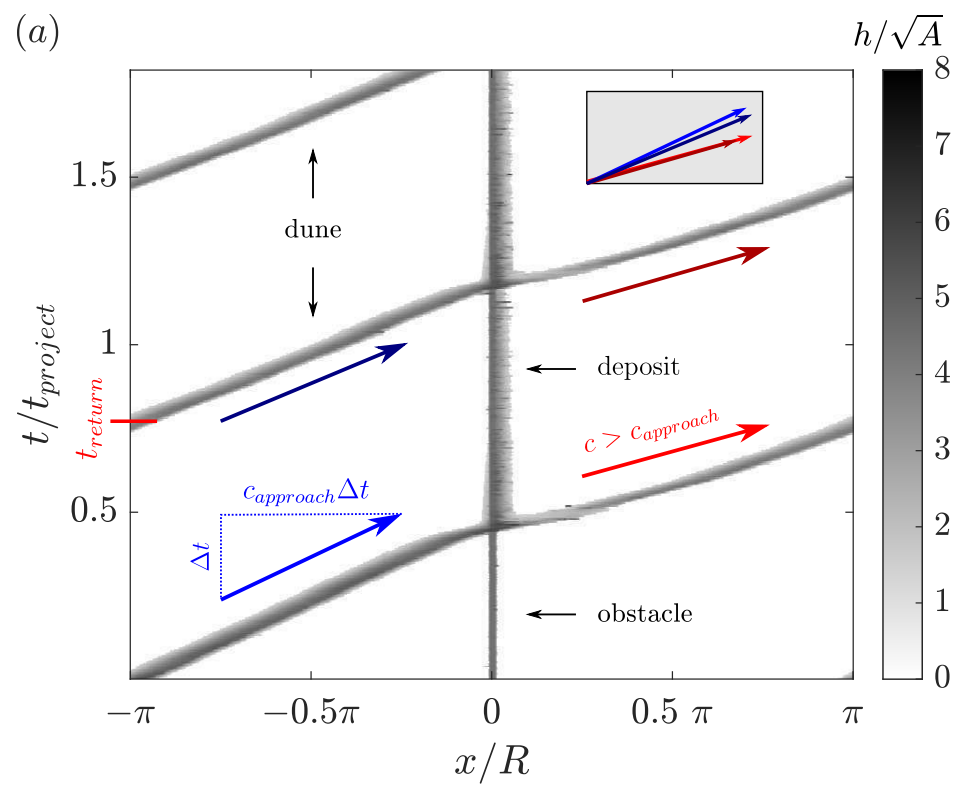

(b)
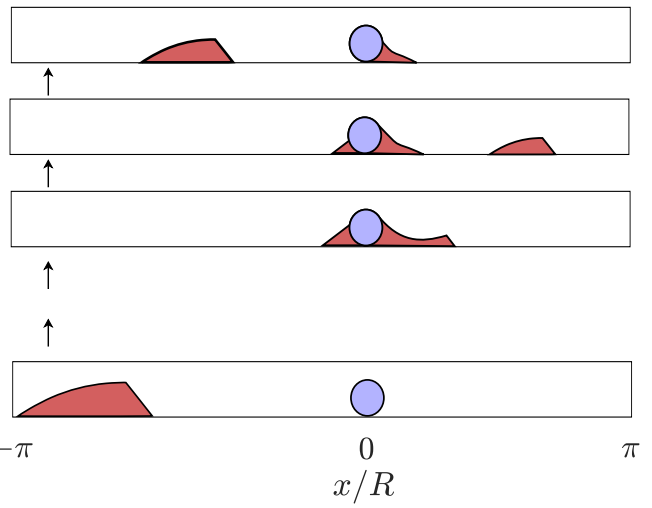

Figure 2. Example experiment with a cylinder of diameter $H_{o}=6 \mathrm{~cm}$ located at $x=0$. (a) The space-time diagram shows a migrating dune interacting with and crossing the solid obstacle. The inset allows us to compare the dune speed $c$ at different points of the experiment (to be precise, the arrows correspond to appropriate linear fits for $x \in[-0.75,-0.25] \pi R$ or $x \in[0.25,0.75] \pi R)$. Note that a vertical arrow would correspond to $c=0$. (b) A schematic showing the progress of a crossing interaction.

DMC-TZ40). It records at a frame rate of $25 \mathrm{~Hz}$ and we use it to visualise the qualitative features of the bed in the vicinity of the obstacle. It is important to note that, due to the battery and memory constraints, this camera is not able to record the experiment continuously.

\section{RESULTS}

\section{A. Qualitative example}

To introduce the physical nature of the dune-obstacle interaction we will now analyse an example experiment, which we will also use as a vehicle to define various quantities of interest. Our selected example is 'Experiment 2' from Table I, lasting 90 minutes. Its entire course is presented in Figure 2 in the form of a space-time diagram. As the domain is periodic, the spatial coordinate $x$ is parametrised with angle $\theta \in[-\pi, \pi)$, so that $x=R \theta$. 

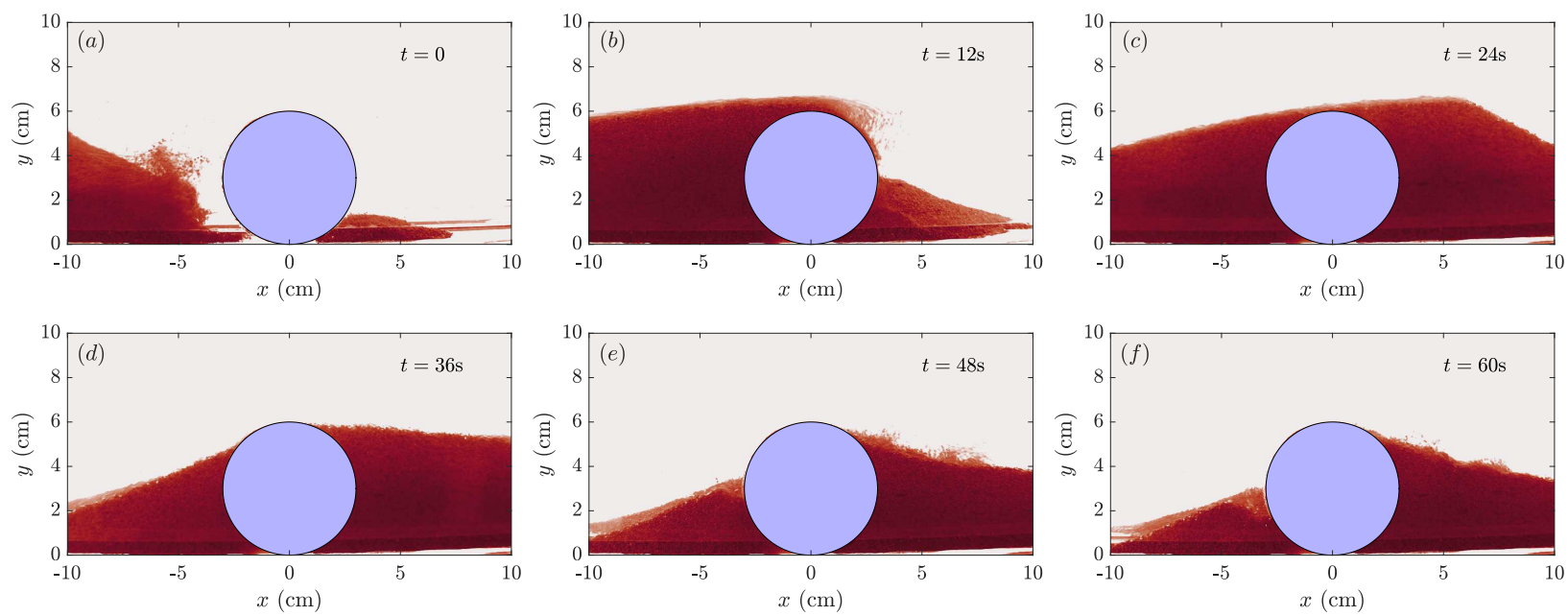

Figure 3. Close-up photographs of a crossing dune-obstacle interaction taken every 12 seconds with a camera mounted to the turntable (camera B in Fig. 1(a)). For visualisation purposes, the obstacle is masked in blue and the rest of the image is printed in false colour. (a) The dune approaches the obstacle. Note that a small amount of sediment swept away from the dune has already accumulated in the downstream recirculation region. (b) The downstream region gradually fills with sediment as the dune 'crosses' the obstacle. (c) At some point, the obstacle is fully buried by the dune. (d-f) The dune's crest moves away from the field of view. Upstream and downstream deposits form and gradually shrink.

The coordinate system is defined so that the centroid of the obstacle is located at $x=0$. At the start of the experiment, the dune is placed at the opposite side of the annular region, at $x=-\pi R$. In this particular experiment, the obstacle is a cylinder of diameter $H_{o}=6 \mathrm{~cm}$ resting on the floor, its axis aligned with the radial direction of the flume. The grey scale of Fig. 2 corresponds to the topographical height $h(x, t)$. Even though the imaging technique does not make a distinction between the dune and the obstacle, we can easily distinguish the fixed obstacle centred at $x=0$.

After initial rearrangement, the dune approaches the obstacle with a close-to-constant migration speed $c_{\text {approach }}=7.4 \mathrm{~m} / \mathrm{h}$. According to classical theory, the migration rate of a quasi-2D steady-state dune is given by

$$
c=\frac{\Delta Q}{H_{d}},
$$

where $\Delta Q$ is the difference in sand (area) flux between the crest and the upstream edge, and $H_{d}$ is the height of the dune $[1,3,16]$. 
The value of $c_{\text {approach }}$ was computed by fitting a straight line to the crest position $x_{c}(t)$, restricted to the interval $x_{c} \in[-0.75 \pi,-0.25 \pi] R$. The crest is the tallest point on the dune and it is unambiguously defined as long as the dune is away from the obstacle. In Sec. II C we will discuss what controls the value of $c_{\text {approach }}$. Throughout this paper we will use

$$
t_{\text {project }}=\frac{2 \pi R}{c_{\text {approach }}}
$$

as a characteristic time scale. It is a projected return time based on the behaviour of the dune on approach, i.e. if it were not for the obstacle, the dune would complete one full revolution around the flume within $t_{\text {project }}$ (in this example $t_{\text {project }} \approx 50 \mathrm{~min}$ ). As we can see, the presence of the obstacle effectively speeds up the dune, so now the dune returns to $x=\pi R$ at $t_{\text {return }} \approx 0.75 t_{\text {project }}$.

At $t \approx 0.45 t_{\text {project }}$, the dune comes into close proximity of the obstacle and a (crossing) interaction ensues. Figure 3 shows close-up photographs of the dune-obstacle collision taken with a co-rotating camera mounted to the turntable (camera B in Fig. 1(a)). For technical reasons, these images correspond to a different realisation of the same experiment and the times indicated in Fig. 3 are not calibrated with Fig. 2. Note that the spatial extent of the $\mathrm{x}$-axis in Fig. $3 \Delta x$ is approximately $20 \mathrm{~cm}$. If it were not for the obstacle, the dune's crest would traverse this extent in $\Delta t=\frac{\Delta x}{c_{\text {approach }}}=97 \mathrm{~s}$, but Fig. 3 shows that in the presence of the obstacle, the same traverse only takes about 30s. The reason for this significant speed-up is partly geometric (the sedimentary layer passes through a contraction) and partly dynamic (the fluid speeding up around the obstacle increases sediment flux). Interestingly, Fig. 3(c) shows that at a certain stage of the dune's crossing the obstacle is fully buried. At this stage the obstacle is dynamically 'cloaked', i.e. for all intents and purposes the bed dynamics is equivalent to that of a bigger dune. Nevertheless, after several seconds, the surface of the obstacle is exposed on the upstream side by the flow again and we can see a particular bed shape forming both upstream and downstream of the obstacle (Fig. 3(d-f)). The upstream deposit shrinks and by consulting Fig. 2 we can verify that after 10 minutes the deposit is completely eroded away, with all the granular material having crossed the obstacle.

The evolution of the downstream deposit is slightly more complicated. Transiently, it forms an elongated sheet stretching from the obstacle to the downstream edge of the dune, but eventually the sheet pinches off and divides into two parts (Fig. 2(b)). The downstream portion of sediment forms an isolated migrating dune and the upstream portion is retained 
in the immediate downstream vicinity of the obstacle, showing that even when all the dune material crosses an obstacle, it is possible for the actual dune to be smaller when it reforms and migrates downstream.

As we can see in the inset in Figure 2, the migration rate of the dune which recovers downstream is significantly larger than $c_{\text {approach }}$, but it decelerates as it moves away from the obstacle. The physical origin of this change is speed is twofold. First of all, after crossing the obstacle the dune is exposed to intense turbulent eddies shed off the upstream obstacle which enrich the sediment flux, i.e. increase the numerator of (2) [4]. Their strength decays away from the obstacle, which is one reason for the dune deceleration. Secondly, the recovery dune is smaller (c.f. the denominator of (2)), as a small amount of sediment is permanently trapped by the obstacle. Nevertheless, as part of the near-obstacle residue is slowly eroded away, it gradually gains mass, which is the other reason for deceleration. The downstream deposit is never completely eroded away, so at the second approach the dune is slightly smaller (and therefore faster) than it was at the first approach. However the deposition after subsequent crossings does not appear to be larger than it was after the first crossing. Therefore, after the second crossing, the dynamics is almost identical to that after the first crossing, so after the second crossing the process would just repeat periodically. In other words, while the first crossing is distinctly different, it has been verified that the $n^{\text {th }}$ crossing is nearly-identical for all $n \geq 2$.

\section{B. Flow measurements}

Apart from the topographical record of the dune-obstacle interaction, we also estimate the mean velocity field $(\bar{U}, \bar{V})$ around each of the obstacles in the absence of the dune. The details of this procedure are explained in Sec. II C. Figure 4 shows the azimuthal velocity field for our example obstacle (Experiment 2). Away from the obstacle, the velocity profile we measure is close to linear, but it is significantly altered in the vicinity of the obstacle. Above the obstacle, there is a significant flow speed-up and both upstream and downstream of the obstacle we can observe zones of depleted momentum. Although the PTV technique is not able to resolve the details of the downstream wake, in the experiment we also observe a clear recirculation bubble forming downstream of the obstacle. In Section III D we will analyse the differences in the flow patterns around different obstacles in more depth. However, before we 


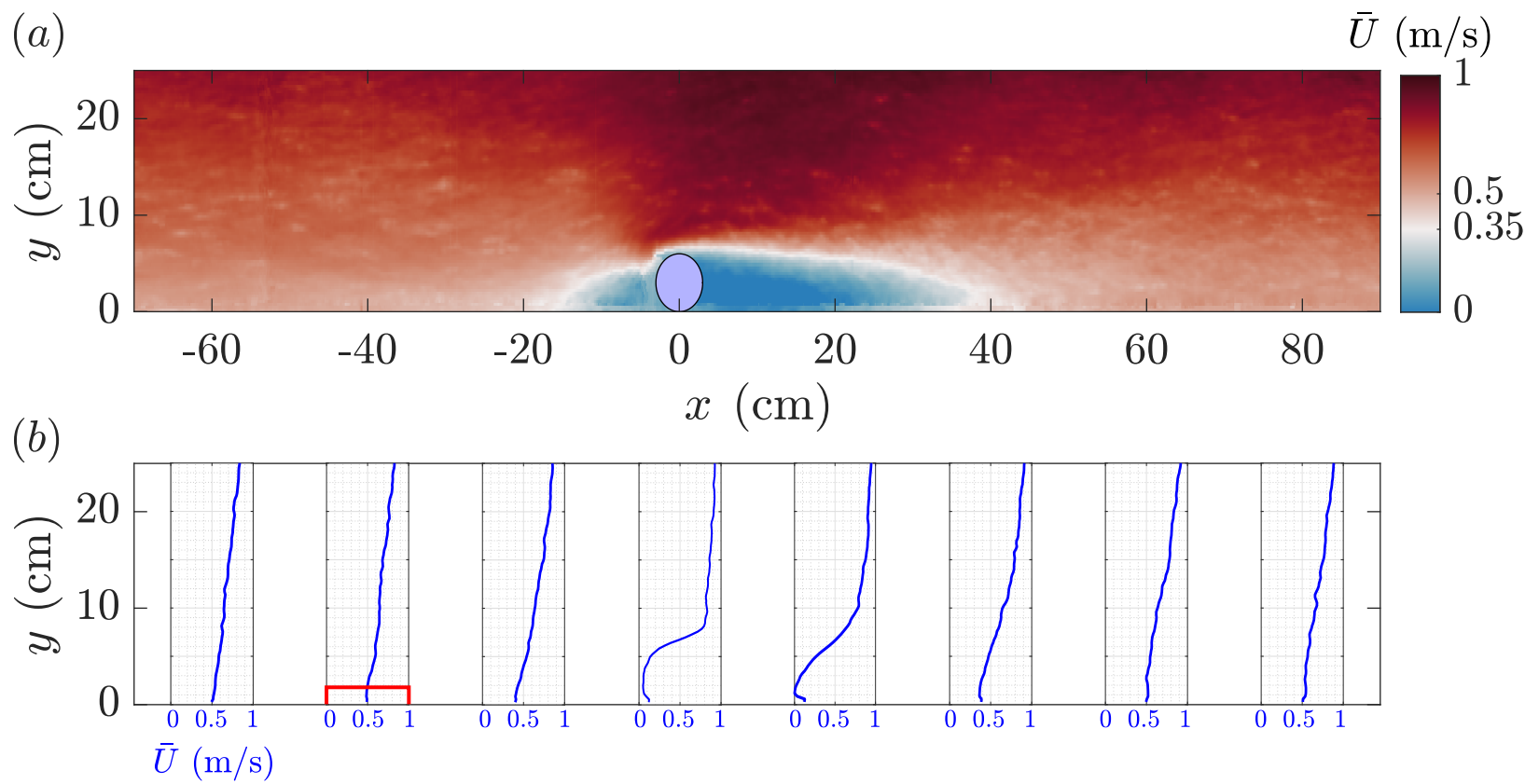

Figure 4. Example PTV measurements. (a) The ensemble-averaged azimuthal velocity $\bar{U}$ around a cylinder with $H_{o}=6 \mathrm{~cm}$. Note the flow speed-up above the obstacle and the momentum deficit in the wake. (b) Each inset shows the velocity profile $\bar{U}(y)$ averaged for $x \in\left[x_{0}-5 \mathrm{~cm}, x_{0}+5 \mathrm{~cm}\right]$, where $x_{0} \in\{-60 \mathrm{~cm},-40 \mathrm{~cm},-20 \mathrm{~cm}, 0 \mathrm{~cm}, 20 \mathrm{~cm}, 40 \mathrm{~cm}, 60 \mathrm{~cm}, 80 \mathrm{~cm}\}$. For the region corresponding to the obstacle, we set $\bar{U}=0$. The red box of size $10 \mathrm{~cm} \times 2 \mathrm{~cm}$ corresponds to the region near $x=-40 \mathrm{~cm}$ where we define $u_{\text {incid }}$.

proceed we need to make an important technical remark about the incident flow upstream of the obstacle.

\section{Incident speed}

We quantify the upstream boundary conditions with incident surface velocity $u_{\text {incid }}$, which is defined as a spatio-temporal average of $\bar{U}$ in a particular wall-attached interrogation window located at a fixed distance upstream of the obstacle (Fig. 4(b)). As the PTV technique is not able to resolve the thin boundary layer due to resolution constraints, $u_{\text {incid }}$ is far from zero. As expected, $u_{\text {incid }}$ is an increasing function of $\Omega_{t o t}$, which is the counterrotation speed of the driving assembly (Fig. 5(a)). However, for fixed $\Omega_{t o t}$ we also see systematic differences between different obstacles. Figure 5(a) shows that $u_{\text {incid }}$ is smaller for the obstacles which are big and sharp (e.g. ridge). This is because the velocity distribution 
$(a)$

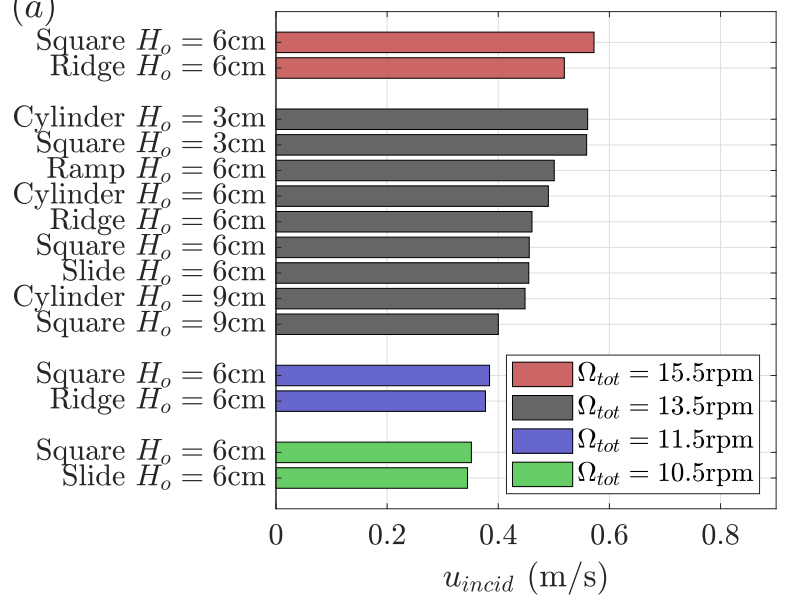

(b)

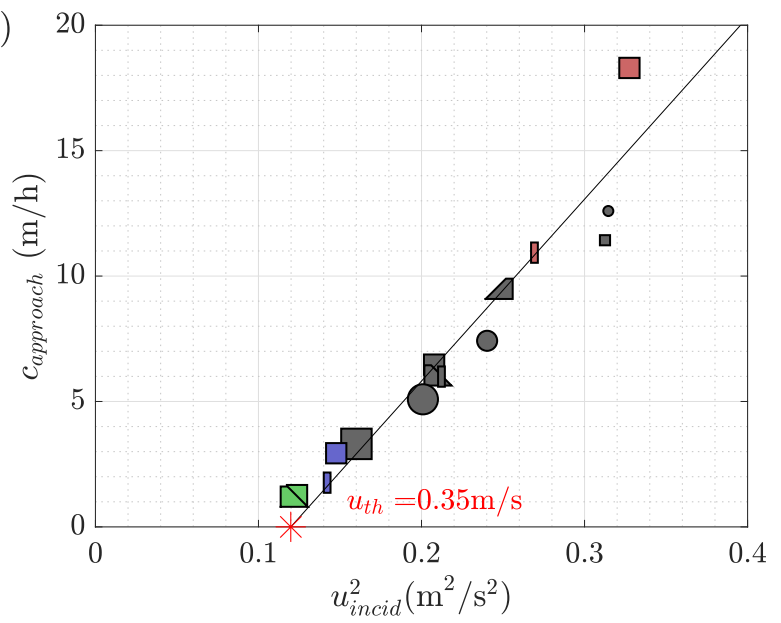

Figure 5. Incident speed. (a) The incident speed measured for different obstacles, under flow conditions defined in Tab. I, but without any sediment in the tank. Note that apart from $\Omega_{t o t}$, $u_{\text {incid }}$ is strongly affected by the size and shape of the obstacles. For fixed $\Omega_{t o t}$, the figure can be interpreted as a ranking of obstacles, from least disruptive (small, blunt), to most disruptive (big, sharp). (b) The incident speed is monotonically related to the dune approach speed. Here we fit an empirical law (4). The symbols in (b) are coloured with the rotation speeds as shown in (a), with symbol shape and size being related to the particular obstacle used in the relevant experiment.

at the bottom wall is not only a function of the velocity prescribed at the top, but also of the surface roughness. Thus, if an obstacle imposes significant form drag, the mean azimuthal velocity will be reduced. In some sense, the effect of a sharp obstacle fixed at the bottom is analogous to having an additional blade rotating in the opposite direction to the flow-inducing driving paddles.

The differences in the incident fluid velocity $u_{\text {incid }}$ explain the differences in dune approach speed $c_{\text {approach }}$. According to formula (2), for fixed dune height the migration rate is directly proportional to the differential sediment flux $\Delta Q$, which in turn is a function of the shear stress exerted on the sedimentary bed. A linearised transport law would assume that sediment flux is proportional to the excess shear stress, which in turbulent flows can be expressed as a quadratic function of appropriate velocities $[2,17]$. Even though $u_{\text {incid }}$ is not necessarily the most appropriate velocity, Fig. $5(\mathrm{~b})$ shows that the differences in $c_{\text {approach }}$ 
between different experiments are indeed well described by an empirical relation

$$
c_{\text {approach }} \propto u_{\text {incid }}^{2}-u_{t h}^{2}
$$

where we fit $u_{t h}=0.35 \mathrm{~m} / \mathrm{s}$. In summary, even though the dune is always of the same size, the approach speed $c_{\text {approach }}$ varies between different experiments, and we can explain the differences by measuring the fluid velocity near the bottom wall.

\section{Crossing-trapping transition}

In Section III A we described a dune-obstacle interaction, where despite some mass reduction, the dune recovered downstream as a coherent migrating structure. This regime, which we refer to as 'obstacle crossing' or simply 'crossing', is one of the two possible outcomes of the dune-obstacle interaction. The alternative will be referred to as 'dune trapping', or simply that the dune is 'trapped'. In this regime, the sediment capture is not partial, but complete, and the dune does not reform downstream.

In this Section, we describe qualitatively the impact of obstacle size (III C 1), obstacle shape (III C 2), and the background flow speed (III C 3) on the outcome of the dune-obstacle interaction (i.e. crossing or trapping). In the next Section (IIID), we will relate these results to the flow structure around the obstacle in a quantitative manner.

\section{Size effects}

The outcome of the dune-obstacle interaction is first and foremost controlled by the size ratio

$$
\eta=H_{o} / \sqrt{A}
$$

As $\eta \rightarrow 0$, the obstacle becomes indistinguishable from the surface roughness, so the dune can cross it almost uninterrupted. Conversely, if $\eta \gg 1$, the obstacle plausibly blocks the motion of the dune, thus trapping all the granular material in the immediate vicinity of the obstacle. That is indeed what we observe for both cylindrical (Fig. 6) and square obstacles (Fig. 7). In Fig. 6/7(a) the height of the approaching dune $H_{d}$ is significantly larger than $H_{o}$, in Fig. 6/7(b) $H_{d} \approx H_{o}$, and in Fig. 6/7(c) $H_{d}<H_{o}$. Nevertheless, the aspect ratio of the dune is not constant, so we will continue to use $\eta$ instead of $H_{o} / H_{d}$. For both cylinders 

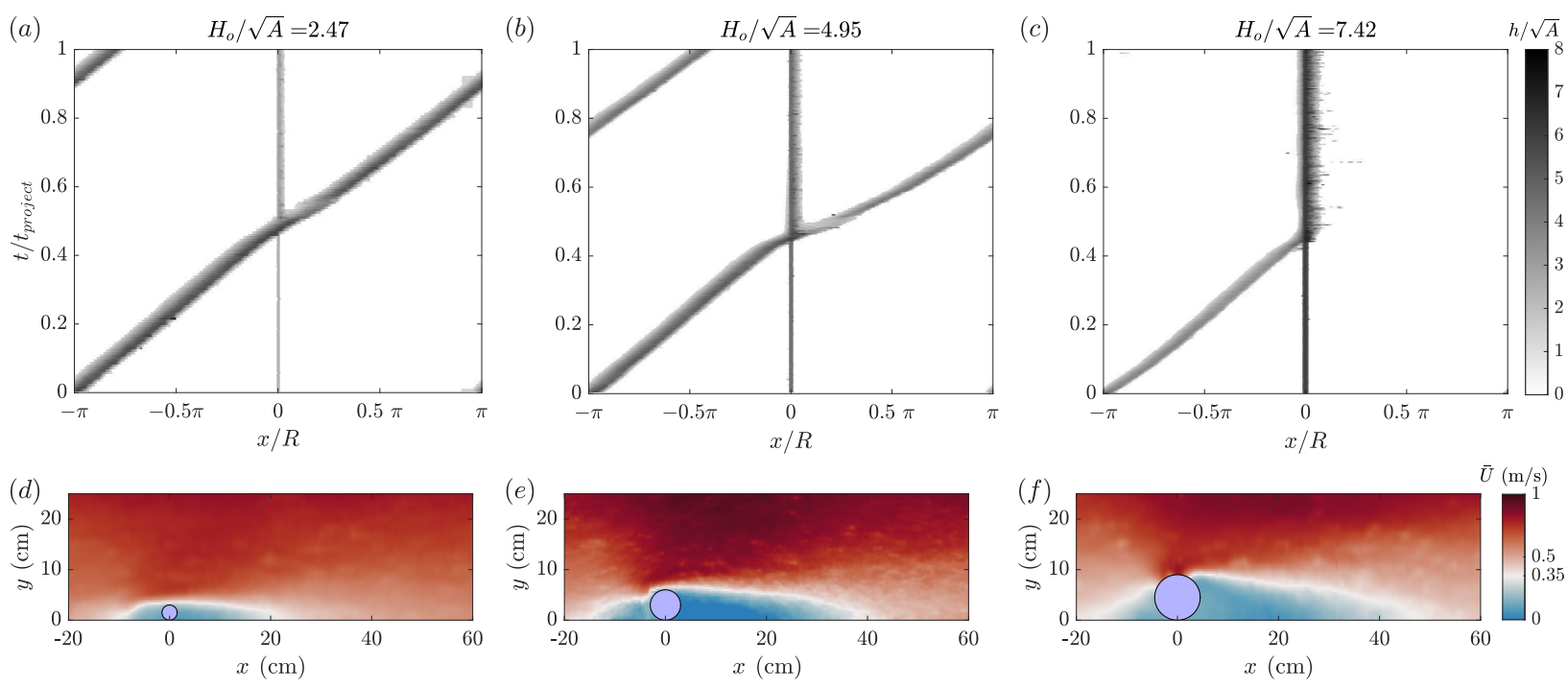

Figure 6. Dune-cylinder interaction. (a) $H_{o}=3 \mathrm{~cm}$ (Experiment 1) - crossing. (b) $H_{o}=6 \mathrm{~cm}$ $\approx H_{d}$ (Experiment 2) - crossing. (c) $H_{o}=9 \mathrm{~cm}$ (Experiment 3) - trapping. (d)-(f) PTV-measured field for the corresponding experiments showing that the size of the wake region increases with $H_{o}$.

and squares, the dune crosses the two smaller obstacles and it is trapped by the obstacle with $\eta=7.42$, as illustrated in Fig. 6c and 7c.

As expected, the smaller the obstacle, the less effect it has on the course of the migrating dune. This can be quantified by looking at the ratio of the actual return time $t_{\text {return }}$ and the projected return time $t_{\text {project }}$. For example, for the small square $\left(H_{o}=3 \mathrm{~cm}\right)$ $t_{\text {return }} \approx 0.9 t_{\text {project }}$ and for the larger square $\left(H_{o}=6 \mathrm{~cm}\right) t_{\text {return }} \approx 0.7 t_{\text {project }}($ Fig. $7(\mathrm{a}-\mathrm{b}))$. As previously noted, in the absence of an obstacle $t_{\text {return }}=t_{\text {project }}$, and small values of the ratio $t_{\text {return }} / t_{\text {project }}$ correspond to large distortions in the projected trajectory.

\section{Shape effects}

Based on the considerations of the previous paragraph, one may postulate the existence of a critical size ratio $\eta_{\text {crit }}$ which delimits the crossing-trapping boundary. We find, however, that $\eta_{\text {crit }}$ also a function of the obstacle shape. For example, by comparing Figures 6 and 7 we can observe that for fixed $H_{o}, t_{\text {return }}$ is smaller for the squares than the cylinders. It indicates that squares are more capable of trapping sediment than cylinders, which suggests that $\eta_{\text {crit }}$ is larger for cylinders.

A more striking example is provided in Fig. 8(a), where the obstacle is a 'ridge' of height 

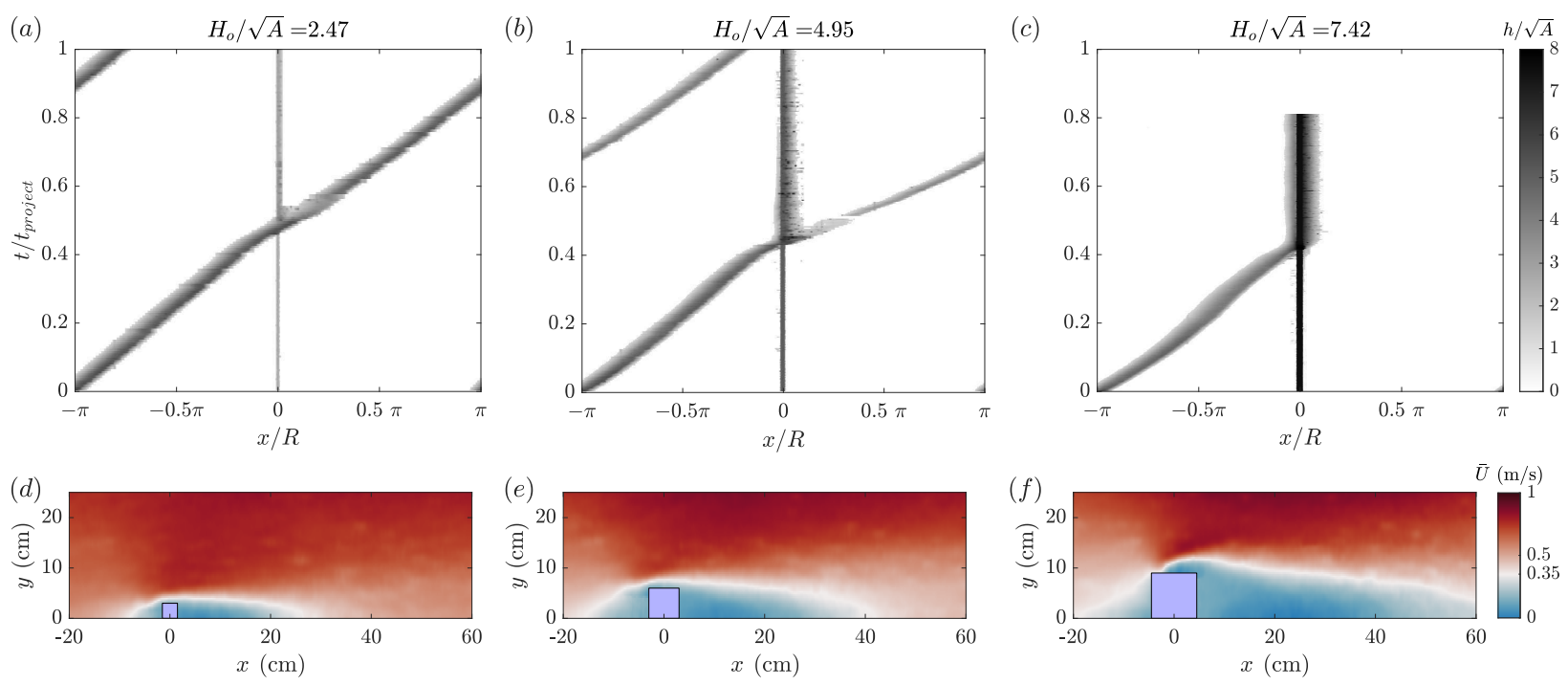

Figure 7. Dune-square interaction. (a) $H_{o}=3 \mathrm{~cm}$ (Experiment 4 ) - crossing. (b) $H_{o}=6 \mathrm{~cm} \approx H_{d}$ (Experiment 7) - crossing. (c) $H_{o}=9 \mathrm{~cm}$ (Experiment 9) - trapping. (d)-(f) PTV-measured field for the corresponding experiments showing that the size of the wake region increases with $H_{o}$ (c.f. Fig. 6).

$H_{o}=6 \mathrm{~cm}(\eta=4.95)$. Although, the dune was able to cross both square and cylindrical obstacles of the same height, it is clearly trapped in the wake of the ridge. This illustrates the important point that trapping does not correspond simply to the granular material piling up on the upstream side of an obstacle. It is also possible that granular material crosses over the obstacle, but is then trapped sufficiently close to the obstacle in the downstream lee so that the dune is not observed to reorganise into a coherent form and migrate away. Interestingly, the subtle interplay between the granular material and the upstream and downstream flow near to the obstacle is illustrated further by the observation that the trapping propensity of the ridge is mitigated by appending to it either an upstream ramp or a downstream slide. Note that for different shapes, the flow structure around the obstacle is different. In particular, Fig. 8(d)-(f) indeed shows that the influence of the ridge, which is the sharpest obstacle, extends further downstream than in the case of either the ramp or the slide, thus making downstream trapping more likely to occur. 

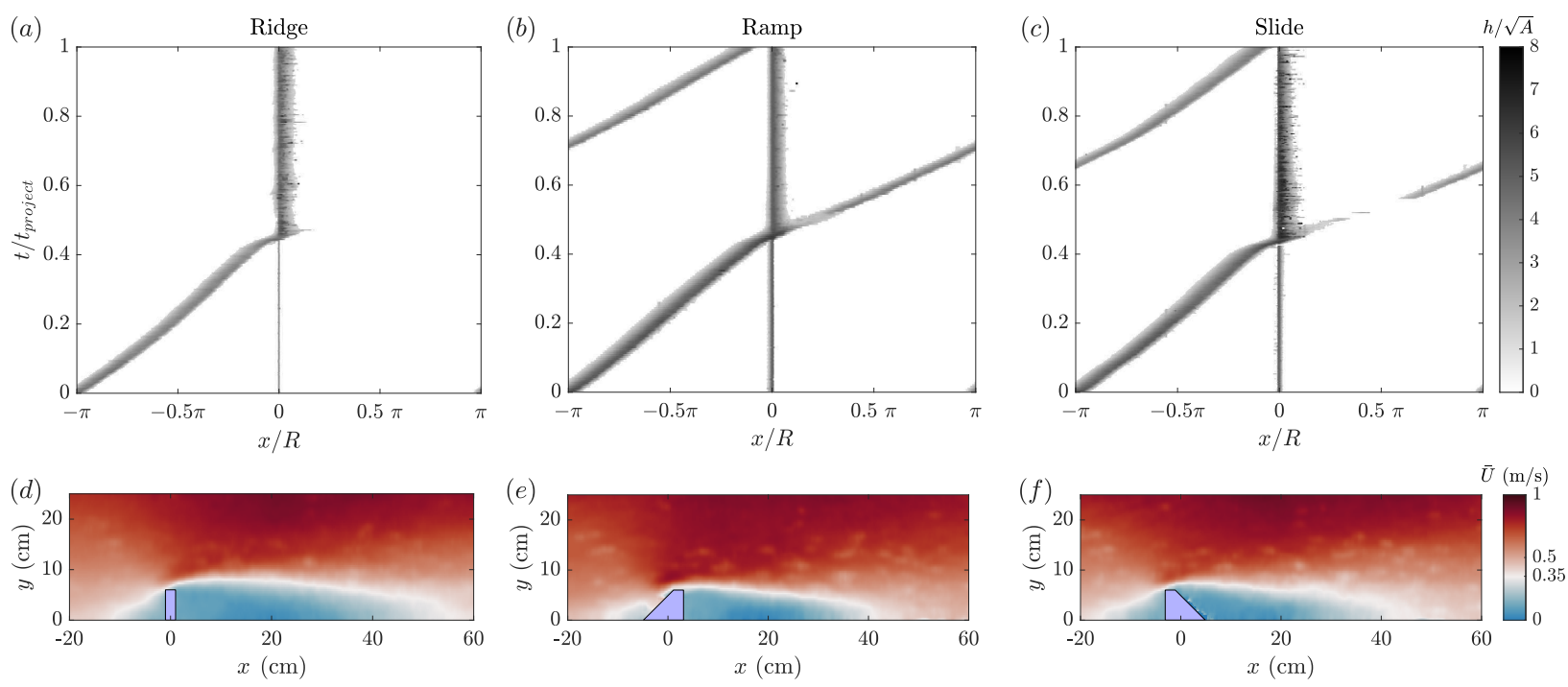

Figure 8. Shape effects. (a) Ridge of height $H_{o}=6 \mathrm{~cm}$ traps the migrating dune (Experiment 11).

(b) The outcome can be changed by appending a ramp (Experiment 13), (c) or a slide (Experiment 14). See Fig. 1(b)) for the definition of the shapes. The gap in data in (c) near $x=0.5 \pi R$ indicates that the transient bedform was so flat that it fell below our noise level $h=1.6 \mathrm{~cm}$. (d-f) The differences between the three experiments can be related to the PTV measurements. The turbulent wake forming downstream of the ridge is larger than the ones behind the ramp or the slide.

\section{Flow speed effects}

Some systematic differences are also observed as we vary $\Omega_{t o t}$, which controls the ambient flow conditions, and therefore the intensity of sediment flux (Fig. 9). Figure 10 shows that for squares with $H_{o}=6 \mathrm{~cm}, t_{\text {return }}$ decreases with the incident speed $u_{\text {incid }}$, i.e. as the sediment transport diminishes, it is more difficult for the dune to cross the obstacle. This effect, however, is surprisingly weak. In particular, within the constraints of our experimental parameters, we have not been able to change the binary outcome of the dune-obstacle interaction. The dune always traverses the square and the slide with $H_{o}=6 \mathrm{~cm}$, and it is always trapped by the ridge of the same height. 

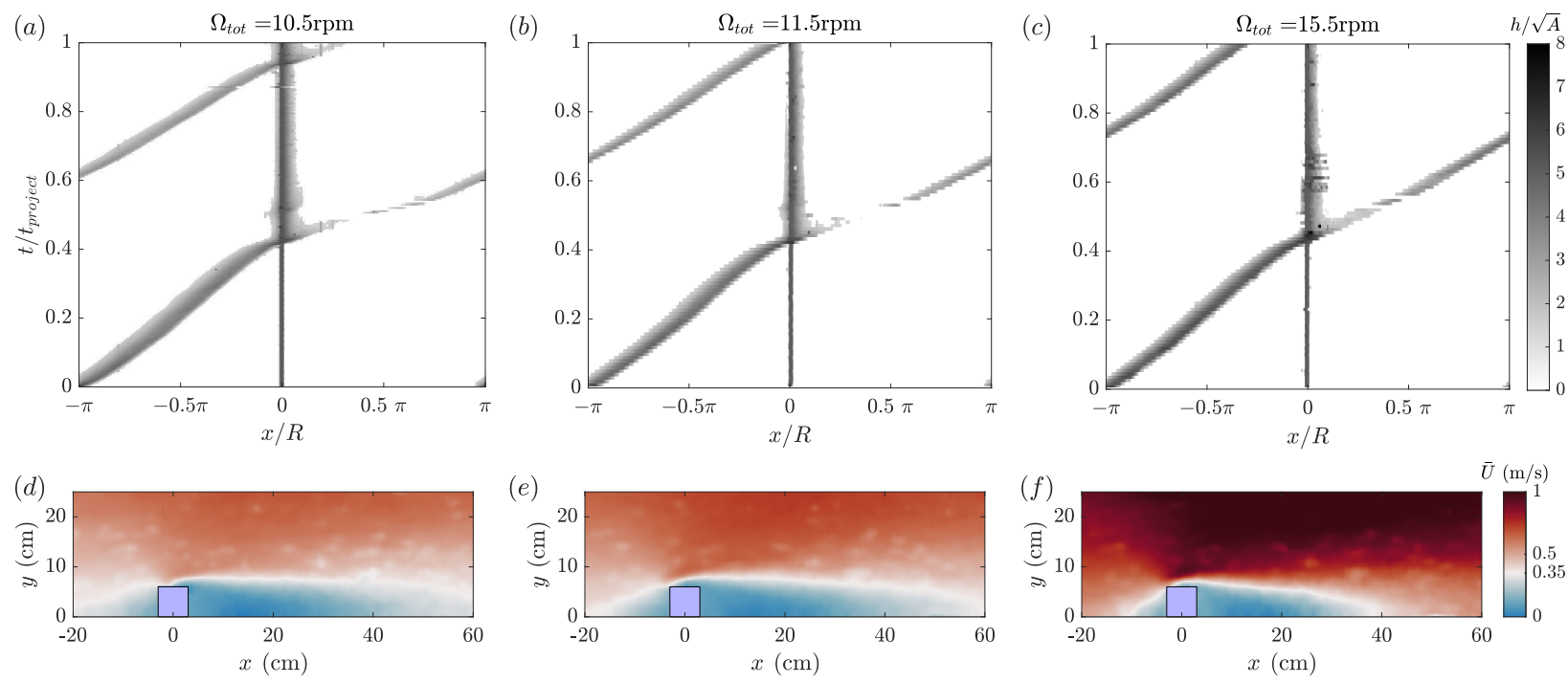

Figure 9. Flow speed effects. In all the panels, the obstacle is a square with $H_{o}=6 \mathrm{~cm}$.

The return time $t_{\text {return }}$ increases as we increase counter-rotation rate $\Omega_{t o t}$ from (a) $10.5 \mathrm{rpm}$ (Experiment 5), through (b) $11.5 \mathrm{rpm}$ (Experiment 6), to (c) $15.5 \mathrm{rpm}$ (Experiment 8). Note that an intermediate case (13.5 rpm) has already been presented in Fig. 7(b). (d-f) PTV measurements confirm that the average azimuthal velocity of the fluid increases with $\Omega_{t o t}$. This increase correlates with the increase in the dune migration rate and so $t_{\text {project }}$ is approximately 14 times larger in panel (a) than in panel (c).

\section{Relating the outcome of a dune-obstacle interaction to the flow structure}

The previous section can be summarised by saying that the outcome of the dune-obstacle interaction depends strongly on the size and shape of the obstacle, and weakly on the background fluid velocity. Now, we will attempt to synthesise these findings by relating them to the flow field measurements.

Our aspiration to explain the outcome of the dune-obstacle interaction from the PTV measurements is motivated by a pragmatic quest for an inexpensive diagnostic which would give an indication about the most likely outcome. In reality, the dynamics is far more complex. In particular, when the dune collides with the obstacle, the velocity field is significantly altered and the course of the interaction is controlled by the mutual feedback between the topography and the fluid flow. Therefore, our PTV measurements, conducted in absence of a dune, almost certainly do not capture all the relevant physics. Nevertheless, as we shall 


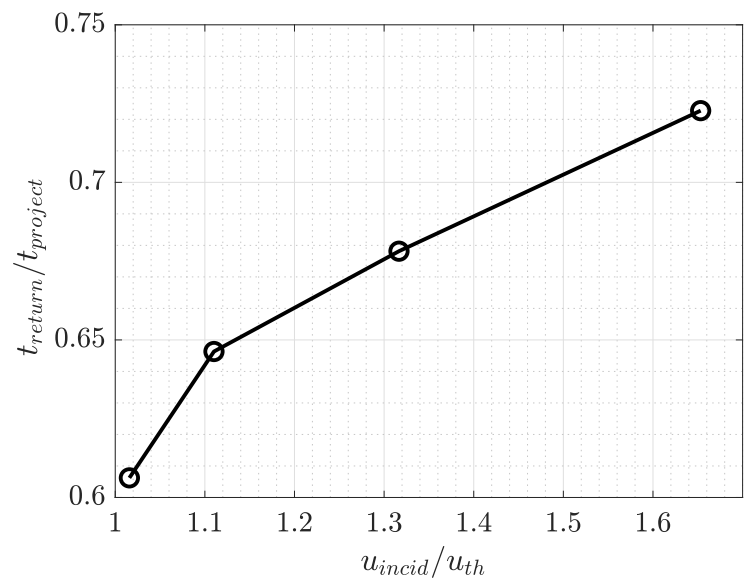

Figure 10. Return time for dune crossing the square with $H_{O}=6 \mathrm{~cm}$ (Experiments 5-8) plotted as a function of the incident flow speed $u_{\text {incid }}$. Small values of $t_{\text {return }} / t_{\text {project }}$ correspond to strong interruption of the migrating dune. Note that $t_{r e t u r n}$ is always lower than $t_{\text {project }}$ and there is an approximate lower bound $t_{\text {return }}>\frac{1}{2} t_{\text {project }}$ (note that by construction the dune collides with the obstacle at $\left.t \approx \frac{1}{2} t_{\text {project }}\right)$.

see, they still encode some highly relevant information.

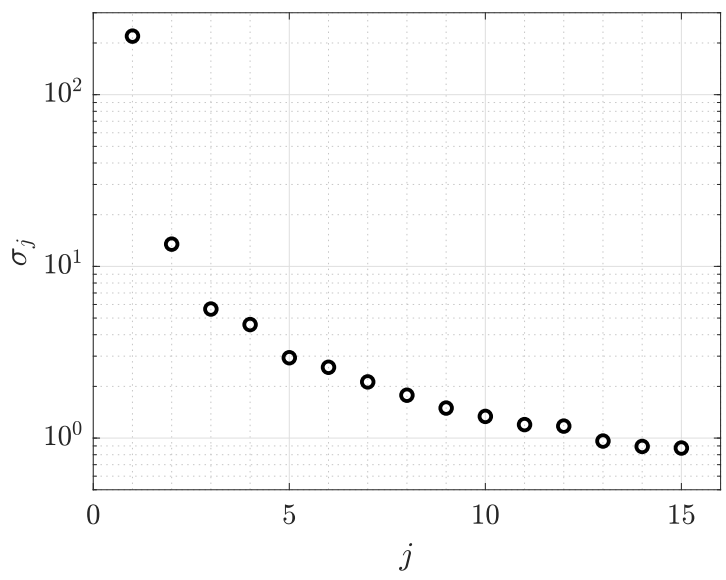

Figure 11. Singular values ordered by magnitude. The leading singular value is larger by an order of magnitude as the data have not been centred.

In lack of an obvious quantitative relationship between the averaged velocity field $(\bar{U}, \bar{V})$ and the dune-obstacle interaction, we shall adopt a data-driven methodology. Specifically, we will employ Principal Component Analysis (PCA) [18]. To this end, for each of the 15 experiments (see Table I), we discretise the azimuthal velocity field $\bar{U}_{i}(x, y)(i=1, \ldots, 15)$ 

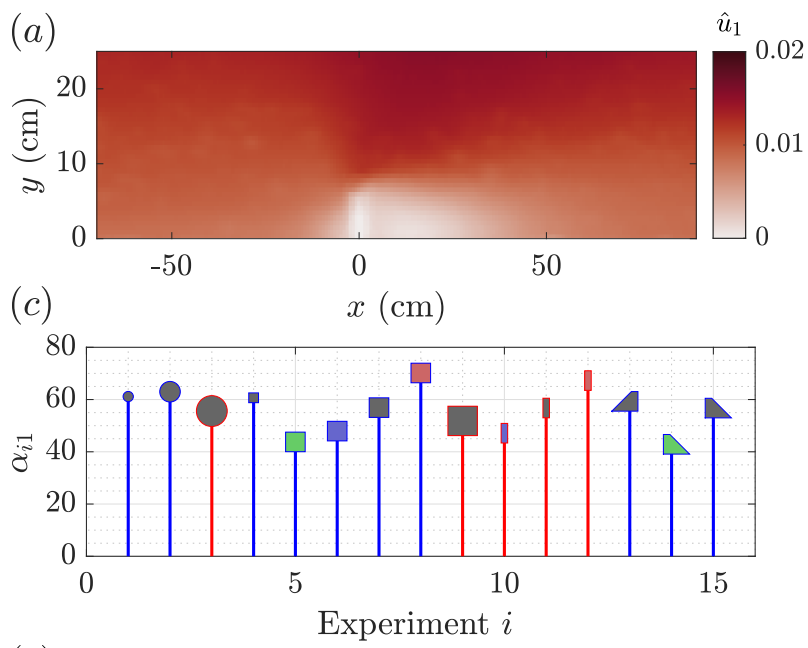

(e)

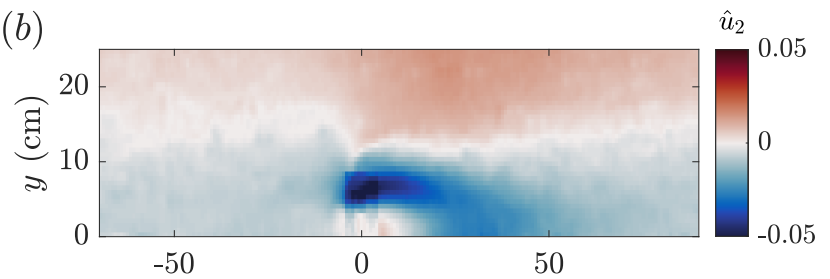

$(d)$

$x(\mathrm{~cm})$

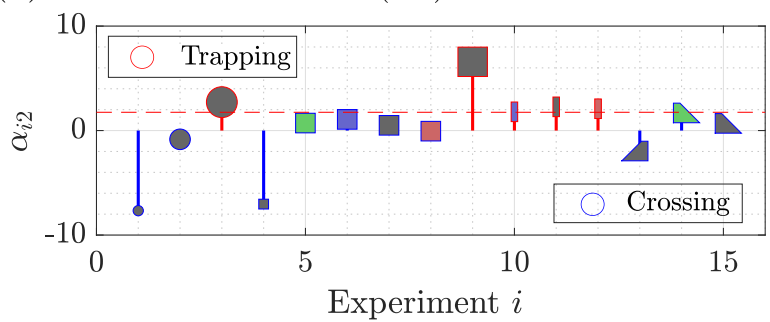

$(f)$
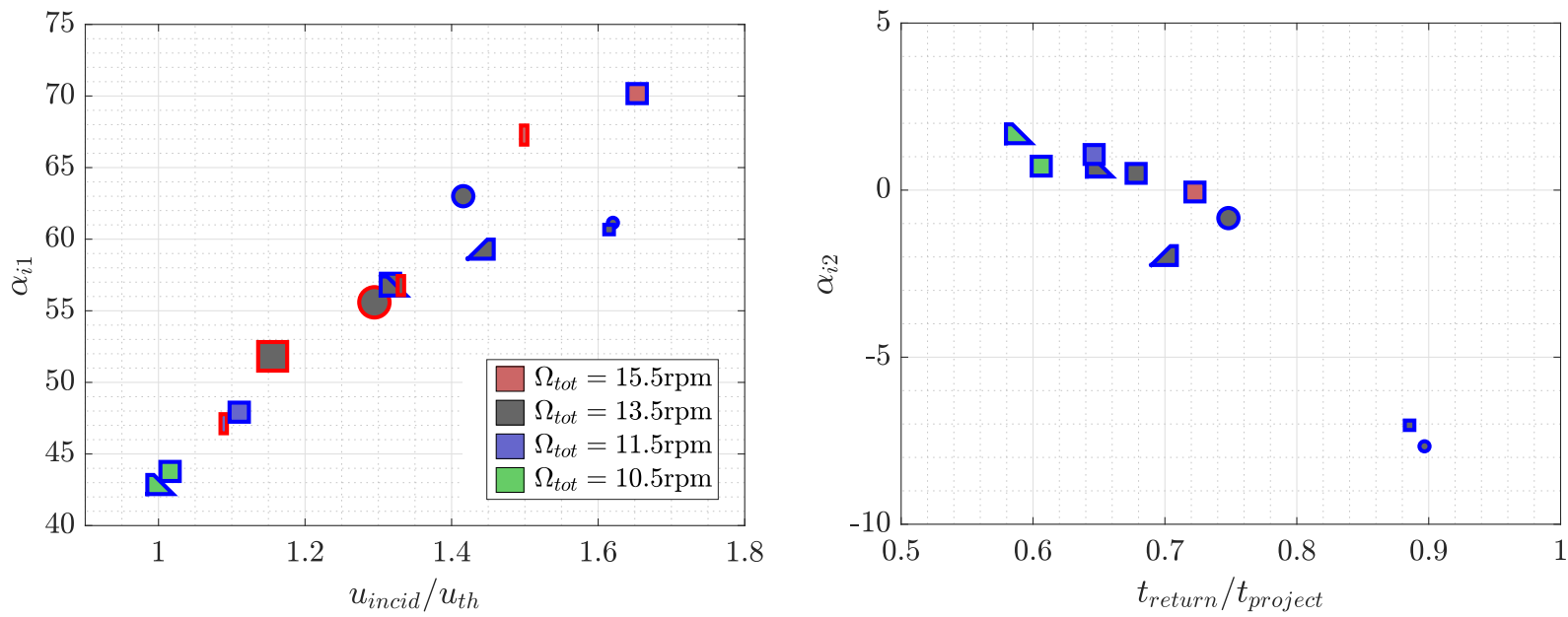

Figure 12. First two principal modes: $\hat{u}_{1}$ (l.h.s. panels) and $\hat{u}_{2}$ (r.h.s. panels). (a) The first principal mode is strictly positive and is closely related to the mean flow field. (b) The second principal mode contains a marked flow structure of negative velocity localised near the obstacle. (c) The weights of the first principal component are all positive. (d) The weights of the second principal component correlate with the outcome of the dune-obstacle interaction with high values for the 'trapping' (red outlined symbols) and low values for the 'crossing' events (blue outlined symbols). The symbols are coloured with the rotation speeds as shown in Fig. 5(a), with symbol shape and size being related to the particular obstacle used in the relevant experiment. (e) The weights for the first principal component correlate with the incident speed $u_{\text {incid }}$. (f) For the 'crossing' experiments, $\alpha_{i 2}$ is negatively correlated with the ratio of the actual return time $t_{r e t u r n}$ to the projected return time $t_{\text {project }}$. Note that $t_{\text {return }}$ is not defined for the 'trapping events'. 
in $x \in[-70,90] \mathrm{cm} \approx[-0.23,0.30] \pi R$ and $y \in[0,25] \mathrm{cm} \approx[0,21] \sqrt{A}$ using a $200 \times 40$ regular grid. Thus, we can treat each velocity field $\bar{U}_{i}$ as a point in a space of dimension $8000=200 \times 40$. Using the whole ensemble of the data points, we perform singular value decomposition to compute the principal components (principal modes) $\hat{u}_{j}(j=1, \ldots, 15)$, so that each data point can be represented as

$$
\bar{U}_{i}=\sum_{j=1}^{15} \alpha_{i j} \hat{u}_{j},
$$

where we refer to $\alpha_{i j}$ as the weights. The principal modes $\hat{u}_{j}$ are ordered based on their energy content, which is quantified by the singular value

$$
\sigma_{j}=\sqrt{\sum_{i=1}^{15} \alpha_{i j}^{2}}
$$

(Fig. 11). We will now focus on the first two most energetic modes.

As the data has not been centred, $\sigma_{1}$ is significantly larger than all the other singular values and the leading first principal component $\hat{u}_{1}$ is closely related to the mean of all the flow fields (Fig. 12(a)). The first principal component $\hat{u}_{1}$ is everywhere positive and the corresponding weights $\alpha_{i 1}$ correlate with the incident speed $u_{\text {incid }}$ (Fig. 12(e)). As noted in section III C 3 , the outcome of the dune-obstacle interaction depends only weakly on the ambient velocity, so it cannot be predicted from either $\alpha_{i 1}$ or $u_{\text {incid }}$.

Of greater interest is the second principal mode $\hat{u}_{2}$, which is a superposition of a simple shear and a localised retardation above and downstream of the obstacle. Interestingly, the associated weights $\alpha_{i 2}$ are strongly related to the outcome of the dune-obstacle interaction. First of all, there is a threshold $\alpha_{2}^{*}$ which perfectly separates the 'trapping' experiments from the 'crossing' experiments (red dashed line in Fig. 12(d)). The 'trapping' events correspond to high values of $\alpha_{i 2}>\alpha_{2}^{*}$ and the 'crossing' events to $\alpha_{i 2}<\alpha_{2}^{*}$. The specific value of $\alpha_{2}^{*}$ is certainly a function of the dune cross-section area $A$ and we hypothesise that $\alpha_{2}^{*}$ would be larger for larger dunes. Moreover, among the 'crossing' experiments, the value of $\alpha_{i 2}$ is negatively correlated with the return time ratio $t_{\text {return }} / t_{\text {project }}$ (Fig. 12(f)).

It has been verified that this trend is insensitive to the details of the discretisation grid. We can also obtain a similar result using the mean-centred version of the PCA or if the modes are constructed using a sub-sample of the data. It is important to emphasise that, as a data-driven method, PCA is completely ignorant of the sand dune context, but our 
result has a plausible physical interpretation. Indeed, it is conceivable that an intense flow structure with negative horizontal velocity (Fig. 12(b)) promotes sediment trapping.

Unsurprisingly, the smallest obstacles (experiments 1 and 4) have the largest value of the ratio $t_{\text {return }} / t_{\text {project }}$, as such obstacles perturb the flow the least. As is apparent in Fig. 12(f), this very small perturbation is associated with a large negative weight for the second principal component. This large negative weight has the main effect of cancelling out the downstream wake structure of the first principal component (as is apparent in Fig. 12(a)), thus leading to a (largely) unperturbed velocity structure.

\section{DISCUSSION}

In this paper we have studied the interaction of a migrating sand dune interacting with a solid obstacle in a paradigmatic quasi-2D setting. Quasi-2D bedforms of our experiments obviously lack the complexity of their 3D analogues, but we believe that understanding quasi-2D interactions is an important first step towards understanding more complicated scenarios. It is also an open question to what extent our results can be extrapolated to aeolian dunes, where the saturation length can be comparable (or indeed greater) than the size of the obstacle.

Our experiments revealed that the dune-obstacle interaction can produce two qualitatively distinct outcomes which we classified as 'crossing' and 'trapping'. We have shown that a dune is more likely to be trapped in the wake of the obstacle if the dune-to-obstacle size ratio $\eta$ is small. We have also found that when $\eta=O(1)$, the outcome of the interaction may depend on the shape of the obstacle. Smooth obstacle shapes (e.g. cylinders) promote crossing and sharp obstacles (e.g. ridges) are more likely to trap the dune. If dune crossing is a desired outcome, this principle should be taken into consideration in designing sub-sea infrastructure. From an academic point of view, the strong dependence of the interaction type on the shape of the obstacle opens an interesting class of optimisation exercises [19]. We have also found that the ambient flow velocity can affect the outcome of the dune-obstacle interaction as well, but in our experiments this effect was relatively weak. In summary, we have found that the dune is more likely to be trapped if the obstacle is large, sharp, and the sediment flux is less intense. We conjecture that this qualitative statement is generally true for a large class of flows and topographies. 
For our specific experiment, we have also managed to develop a quantitative diagnostic whereby the outcome of the dune-obstacle interaction can be predicted from the flow structure around the obstacle in absence of the dune. Specifically, we performed a modal PCA-based analysis of our data and we have found an interesting correlation between the second principal mode and the outcome of the dune-obstacle interaction. This finding affirms that it is indeed possible to predict the outcome of the dune-obstacle interaction with some confidence by looking at the mean velocity in the absence of sediment. Moreover, it shows the power of the spectral analysis and the importance of the flow structure details near the crest and downstream of the obstacle. The details of our analysis are somewhat specific to the boundary conditions of our set-up, but by performing similar studies, e.g. for dunes immersed in a turbulent boundary layer, one may hope to build a predictive library which encompasses a wide class of geologically relevant cases. This task could be greatly aided by numerical simulations and we would like to hope that the experiments of this paper could be used as a validation benchmark.

\section{ACKNOWLEDGMENTS}

KAB acknowledges the support of a sponsored PhD studentship from Schlumberger Cambridge Research. NMV is supported by a Royal Society University Research Fellowship URF/R1/191332. The authors thank Professor Stuart Dalziel and the technical team of the GK Batchelor laboratory Ewas well as Dr Paul Jarvis.

[1] F. Charru, B. Andreotti, and P. Claudin, "Sand Ripples and Dunes," Annual Rev. of Fluid Mech. 45, 469-493 (2013).

[2] B. Andreotti, Y. Forterre, and O. Pouliquen, Granular Media: Between Fluid and Solid (CUP, 2013).

[3] K. Kroy, G. Sauermann, and H. J. Herrmann, "Minimal Model for Sand Dunes," Phys. Rev. Lett. 88, 054301 (2002).

[4] K. A. Bacik, S. Lovett, C. P. Caulfield, and N. M. Vriend, "Wake induced long range repulsion of aqueous dunes," Phys. Rev. Lett. 124 (2020). 
[5] W. R. Assis and E. M. Franklin, "A comprehensive picture for binary interactions of subaqueous barchans," Geophys. Res. Lett. 47, GL089464 (2020).

[6] P. Vermeesch, "Solitary wave behavior in sand dunes observed from space," Geophys. Res. Lett. 32.22 (2011).

[7] P. Hersen and S. Douady, "Collision of barchan dunes as a mechanism of size regulation," Geophys. Res. Lett. 32 (2005).

[8] N. Endo, K. Taniguchi, and A. Katsuki, "Observation of the whole process of interaction between barchans by flume experiments," Geophys. Res. Lett. 31 (2004).

[9] R. D. Lorenz and J. R. Zimbelman, Dune Worlds, How Windblown Sand Shapes Planetary Landscapes (Springer-Verlag, 2014).

[10] L. Bruno, M. Horvat, and L. Raffaele, "Windblown sand along railway infrastructures: A review of challenges and mitigation measures," Journal of Wind Engineering \& Industrial Aerodynamics 177, 340-365 (2018).

[11] W. He, N. Huang, B. Xu, and W. Wang, "Numerical simulation of wind-sand movement in the reversed flow region of a sand dune with a bridge built downstream," The European Physical Journal E 41 (2018).

[12] A. A. A. Alghamdi and N. S. Al-Kahtani, "Sand control measures and sand drift fences," J. Perf. Constr. Facil. (2005).

[13] P. L. Barnard, D.M. Hanes, D. M. Rubin, and R.G. Kvitek, "Giant sand waves at the mouth of San Francisco Bay," Eos, Transactions American Geophysical Union 87, 285-289 (2006).

[14] A. W. Baar, J. de Smit, W.S.J. Uijttewaal, and M.G. Kleinhans, "Sediment transport of fine sand to fine gravel on transverse bed slopes in rotating annular flume experiments," Water Resour. Res. 54 (2018).

[15] S. B. Dalziel, "Decay of rotating turbulence: some particle tracking experiments," Applied Scientific Research 49, 217-244 (1992).

[16] B. Andreotti, P. Claudin, and S. Douady, "Selection of dune shapes and velocities part 1: Dynamics of sand, wind and barchans," The European Physical Journal B 28, 321-339 (2002).

[17] J. F. Kok, E. J. R. Parteli, T. I. Michaels, and D. B. Karam, "The physics of wind-blown sand and dust," Reports on Progress in Physics 75, 106901 (2012).

[18] S.L. Brunton and J.N. Kutz, Data-driven Science and Engineering (CUP, 2019).

[19] M. Horvat, L. M. Horvat, S. Khris, and L. Raffaele, "Aerodynamic shape optimization of 
barriers for windblown sand mitigationusing CFD analysis," Journal of Wind Engineering \& Industrial Aerodynamics 197 (2020). 